\title{
Large area amorphous silicon photodiode arrays for radiotherapy and diagnostic imaging
}

\author{
L.E. Antonuk, J. Yorkston and J. Boudry \\ Department of Radiation Oncolexgy, Unit ersing of Michigan. Ann Arbor, MI 48109, USA
}

\section{M.J. Longo}

Departmeme of Phẹsics, Unic ersity of Michisen. Ann Arbor, MI 4\$109. USA

\author{
R.A. Street \\ Xirox. Palo Alto Reseurch Center. Pule Alto, CA 94304, USA
}

\begin{abstract}
Amorphous silicon imaging devices consisting of two-dimensional pixel arrays of photodiodes and field effect transistors can now be fabricated over areas as large as $30 \mathrm{~cm}$ by $30 \mathrm{~cm}$. Such imagers can offer considerable advantages for real-time radiotherapy megavoltage and diagnostic $X$-ray imaging applications. The design. operation, and advantages of such imagers are discussed. and sensor signal data are presented.
\end{abstract}

\section{Introduction}

Real-time imaging with gamma-ray and X-ray radiation has become a commonplace occurrence in numerous medical procedures. In external beam cancer therapy various techniques have been developed over the last 10 ycars in an attempt to provide a clinically practical means of performing real-time imaging of the megavoltage (") $\mathrm{Co}, 3$ to $50 \mathrm{MV}$ ) photon treatment beams [1-9]. This is motivated by the desire to maximize the delivered dose to the intended treatment volume while minimizing the dose to surrounding tissuc. During treatment, the radiation is collimated in order to allow the dose distribution to conform to the shape of the treatment volume. Development of a means to verify the setup of the patient with respect to the treatment beam immediately before irradiation. called portal localization, and/or during irradiation. called portal verification, would allow a reduction in the margins of the treatment volume around the target volume. This would help permit treatment to higher doses.

In diagnostic imaging. the development of image-intersifier systems over the last 30 years has resulted in the availability of high quality real-time images using diagnostic quality $\mathrm{X}$-ray beams ( $\sim 30$ to $120 \mathrm{kVp}$ ). For the most part, these have been used to provide qualitative information for general fluoroscopy, chest and cardiac imaging, angiography and digital subtraction angingraphy. More recently, considerable interest has developed in extracting more quantitative information such as blood volume flow.

In both radiotherapy and diagnostic applications. currently available imaging instrumentation imposes significant constraints and limitations. However, recent progress in the development of hydrogenated amorphous silicon $(\mathrm{a}-\mathrm{Si}: \mathrm{H})$ scnsors and transistors now offers the prospect of large-area, flat-panel, real-time imaging arrays which would climinate many of these restrictions. In this paper the design and operation of a multi-element amorphous silicon detector array (called MASDA or MASDA array) is presented. The specific advantages of such arrays for radiotherapy and diagnostic imaging are outlined. Signal data obtained with radiotherapy beams and an a-Si:H sensor are presented, followed by a discussion of the prospects for the development of such arrays.

\section{Design and operation of the MASDA imager}

Fig. 1 contains a schematic diagram of the general layout of the MASDA array being developed by our group. The sensing elements are light-sensitive photodiodes. Fach photodiode consists of a bottom metal contact followed by a p-doped. an intrinsic, and an $\mathrm{n}$-doped a-Si:H layer ( $\mathrm{p}-\mathrm{i}-\mathrm{n})$, with a top transparent metal contact (typically Indium-Tin-Oxide, ITO). Either a $p-i-n$ or an $n-i-p$ configuration is possible with the primary contributor to the collected signal being 


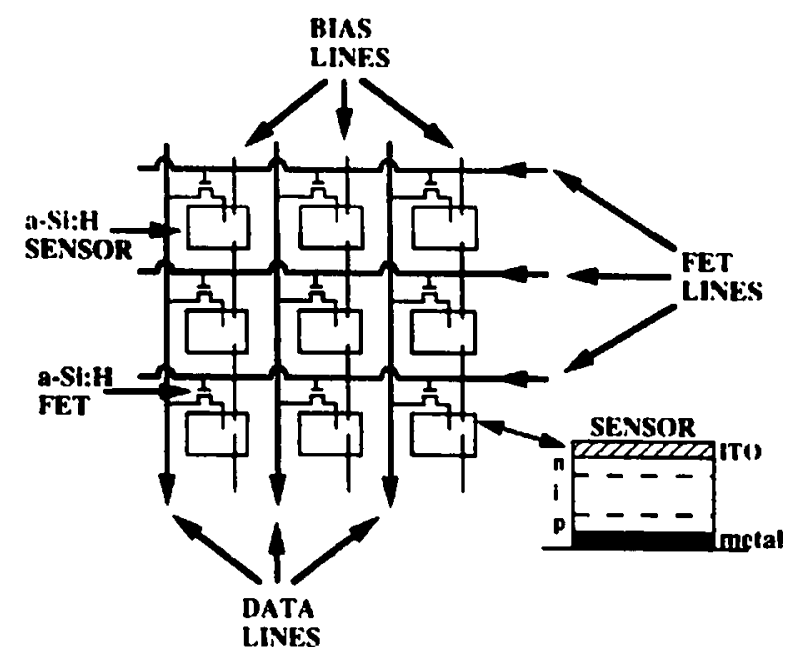

Fig. I. Schematic diagram. not to sale. of the layout of a MASDA array along with the construction of an individual p-i-n sensor.

holes and electrons respectively when light enters through the ITO. The $n$ and $p$ layers range in thickness from 10 to $100 \mathrm{~nm}$ while the $i$ layer nced only be $\sim 1$ $\mu \mathrm{m}$ or greater in order to absorb all incident visible wavelength photons. The sensors are arranged in a regular two-dimensional pattern and each sensor is connected to an adjoining data line by means of an a-Si: $\mathrm{H}$ ficld effect transistor (FET). The gate electrode of each FET is connected to a FET control line. Each sensor and its associated FET constitute an imaging pixel. For an array with $n$ by $n$ pixcls arranged in a square. there are $n^{2}$ sensors. $n$ data lines. and $n$ FET gate control lines. Each data line and FET control line terminates at an independent contact pad at the periphery of the array. Finally. a reverse bias voltage is applied to each sensor by means of a network of bias lines.

The principle of operation of the imager is illustrated in fig. 2. The object to be imaged is placed between the radiation source and the imager. Mega-

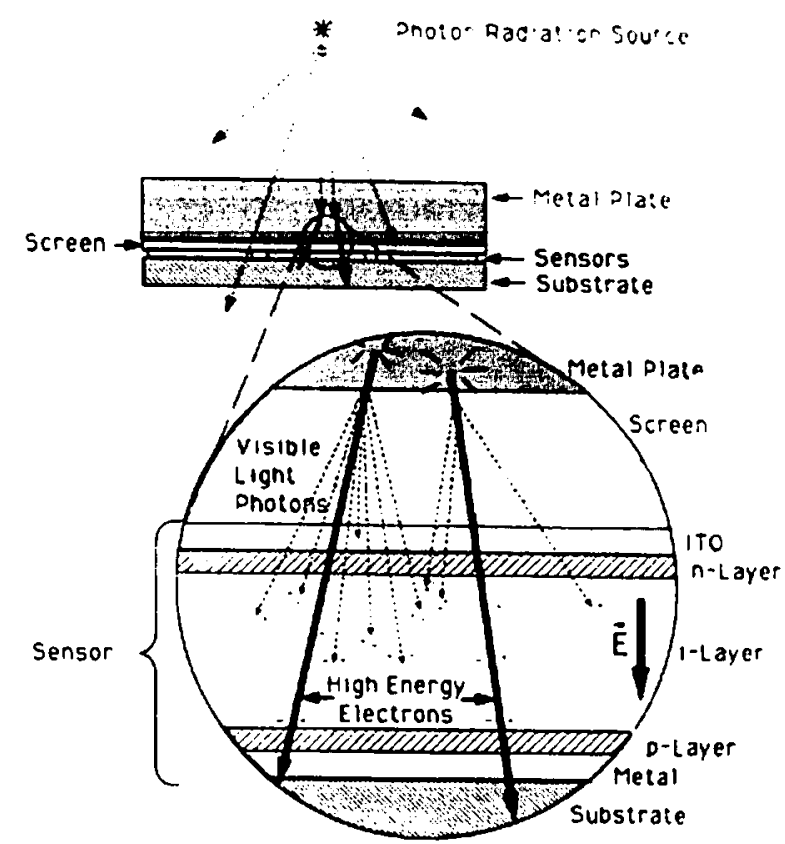

Fig. 2. Diagram illustrating the interaction of high encrgy photon and electron and visible light quanta with a MASDA imager.

voltage or diagnostic $\mathrm{X}$-rays which pass unattenuated through the object interact with the imager creating high energy electrons which generate light as they pass through a phosphor screen For megavoltage radiation the low cross sections associated with the high energy photons means that a metal plate (typically $-1 \mathrm{~mm}$ of copper) must be present to provide sufficient interactions. For diagnostic energies. the cross sections are considerably higher. and conscquently the interaction. can take place in the screen itself with no need for this metal plate. The passage of these secondary high energy electrons through the screen creates visible light photons. Those photons which emerge from the screen and pass into the $i$ layer of the photodiodes are completely absorbed. primarily creating electron-hole pairs

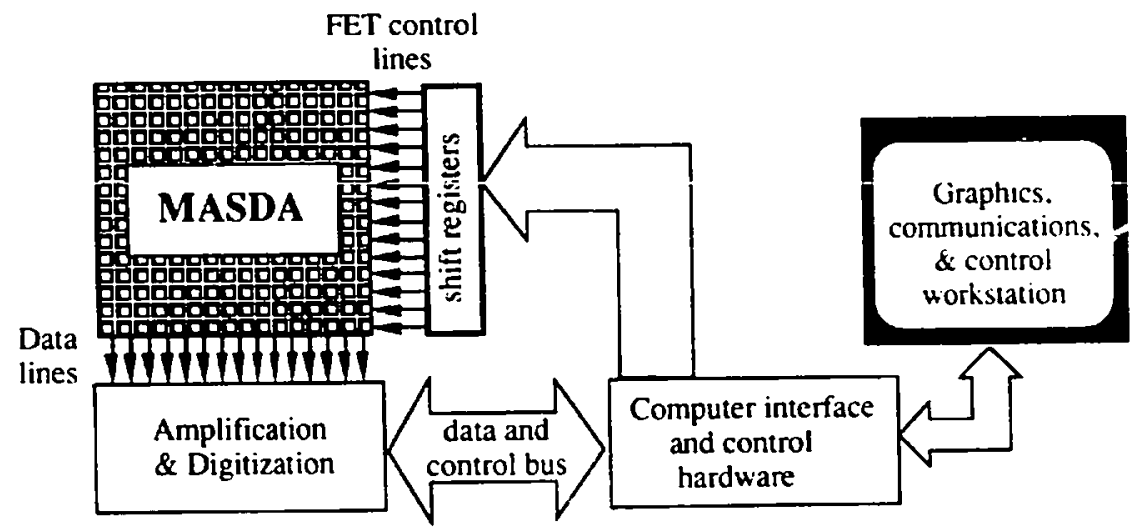

Fig. 3. Block diagram showing the various components of the entire MASDA imager. 
which drift under the influence of the applied electric field.

A block diagram of the exsential elements of a MASDA imager is shown in fig. 3. A graphics. communications, and control workstation sends conmands 10 and receives imaging information from the peripheral clectronics surrounding the MASDA array. The voltuges on the FET control lines may be manipulated by means of digital shift registers. When these control lines are held negative, the FETs are nonconducting and the generated signal accumulates in the sensors. During readout the FETs are made conducting, one row at a time, by driving the FET line bias positive. thereby allowing the charge accumulated in the sensors to propagate to the adjoining data lines. Signals from the data lines are amplified, multiplexed, digitized, and forwarded to the control workstation for further image processing.

\section{Advantages for radiotherapy and diagnostic imaging}

Reported real-time megavoltage imagers include camera-fluoroscopy systems [1-6]. a scanning liquidionization chamber [7]. and linear scanning diode arrays $[8,9]$. Only the fluoroscopic imagers (and the MASDA imager) sense the entire radiation field simultaneously. Like the MASDA imager, the fluoroscopic imagers use a metal plate and phosphor screen. The light is focused onto the sensing plane of a camera by means of a reflecting mirror [1-5] or fiber-optic light pipes [6]. In both cases only a very small fraction of the light emitted from the screen. less than $0.1 \%$ ', for the mirror imagers, actually reaches the camera target and is converted to useful signal. The relative number of quanta for cach stage of a calmera-fluoroscopy (mirror) imager has previously been published [10] and is illustrated in fig. 4. While these calculations apply in the ecro spatial frequency limit, for nonecro spatial irequencies the relative number of light quanta detected by the camera actually falls below the number of high energy electrons released from the metal plate. Under these conditions, the stage limiting the quantum noise for the camera-fluoruscopy imager is the number of light quanta detected by the camera and not the number of electrons released from the metal plate [5]. This results in less than optimal imagc quality. The performance of the MASDA imager, also shown in fig. 4, is the same up to the number of light quanta emitted from the phosphor sereen. Thereafter however, the light collection and conversion efficiency is very high $(-50 \%)$. Consequently, the quantum noise-limiting stage is the number of electrons released from the metal plate, and thus onc anticipates that image quality from the MASDA imager will be superior to that of present camcra-fluoroscopy imagers.

In addition, a MASDA imager would offer several other important advantages for radiotherapy. As the substrate on which the a-Si: $\mathrm{H}$ is grown is typically less than I mm thick. the total imager including integrated circuits for the readout electronics need be no thicker than a film cassette $(\sim 1.5 \mathrm{~cm})$. Such a thin profile would make the device very practical in a clinical setting as it offers minimal obstruction to the motions of the treatment machine and the patient support table. Sccondly, a-Si:H sensors and FETs have

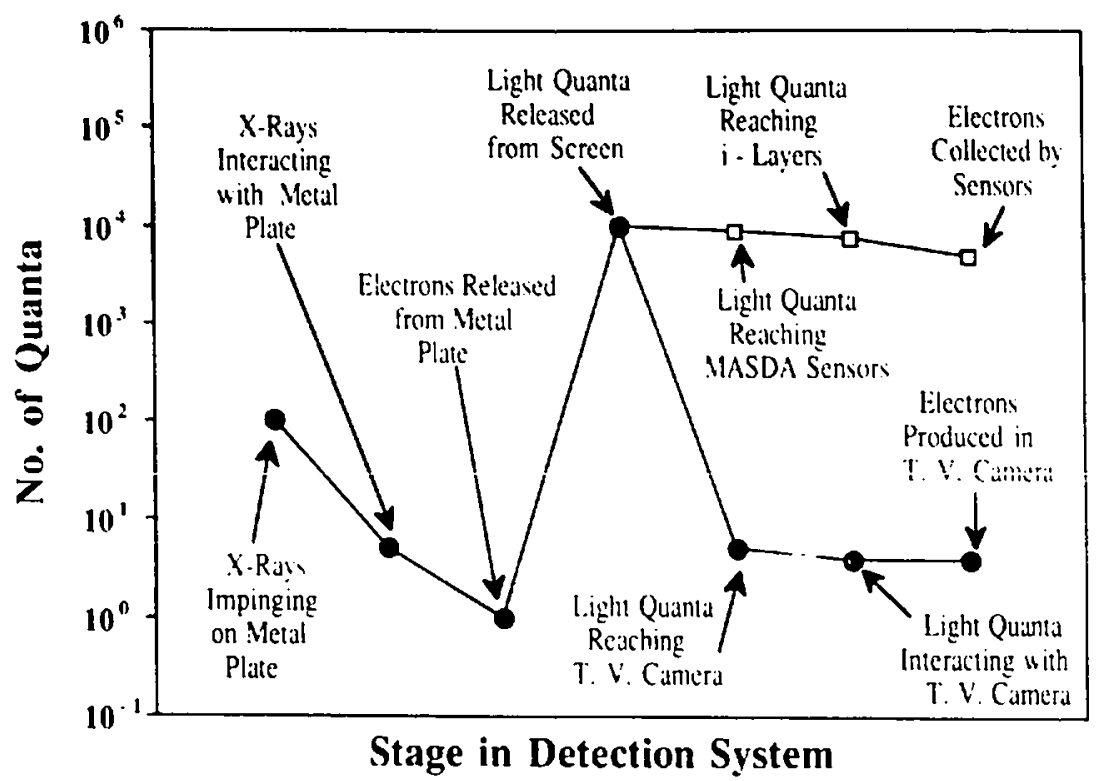

Fig. 4. Diagram showing the relative number of quanta at every stage in a camera-fluoroscopy (solid dots) and a MASDA (open squares) radiotherapy imager. The camera-fluoroscopy data are from ref. [10]. 
demonstrated very high resistance to radiation damage $[11,12]$. This is important in this application as the imager would receive roughly $3 \times 10^{\prime \prime}$ cGy per year during routinc clinicisl use.

Most real-time diagnostic $X$-raly imiging is presentls perlormed using various image-intensitier (II) systems. While these represent a highly developed technology. MASDA-type imagers offer several distinct advantages. Firstly, the hulkiness of II systems often necessitates complex. costly, and cumbersome mechanical supports which can impede the clinician. By comparison. the mechanical support for a thin MASDA imager could be far simpler. Such imagers could even be built into the patient support tables themselves. Secondly. image contrast from II systems is reduced as a result of veiling glare originating from light scatter. A MASDA imager would eliminatc this effect. thereby improving image quality. Finally, II systems suffer geometric distortions. called pincushion distortion. in the image. This originates from the curved surface of the photocathode which serves to enhance the electron optics of II systems. Pincushion distortion would be automatically eliminated with a MASDA imager. thereby facilitating quantitative geometric analysis of the images.

\section{Signal measurements with an a-Si: $\mathbf{H}$ photodiode}

Optimal design of the arrays and readout electronics requires knowledge of the signal sizes gencrated in the a-Si:H sensors. Such measurements have been performed for radiotherapy beams with a $p-i-n$ photodiode of the same design as those used for the arrays. The photodiode had a sensitice surface area of $\$ 3.5 \mu \mathrm{m}$ by $8.35 \mu \mathrm{m}$. $n$ and $p$ layers of $50 \mathrm{~nm}$. an $i$ layer of $1 \mu \mathrm{m}$. and a leakage current of -2 DA at $1.5 \%$. Two phusphor screens were used: (1) a Kodak L.:ince Fast B screitn with $133 \mathrm{mg} / \mathrm{cm}^{2}$ of gadolinium oxysulfide. (id, $, 0, S:$ Th, with a number of discrete emiscion walelengths. the larges of which is at $545 \mathrm{~nm}$, and (2) a Dupont Cronex Lightning Plus screen with 86.8 $\mathrm{mg} / \mathrm{cm}^{2}$ of calcium tungstatc, CaWO $\mathrm{O}_{4}$, with a continuous spectral emission centered at $\mathbf{4 3 0} \mathrm{nm}$. The Lanex screen is one typically used in radio therapy imaging. while both screcns arc used for various diagnostic imaging applications.

Radiotherapy signal measurements were performed at 6. 10., and 15 MV on Varian Clinac 18(0) and Clinac is treatment machines which deliver radiation in -5 $\mu$ s long pulses. The measurement technique is the same as that used earlier on sensors of different construction [13]. The measurements were taken at $100 \mathrm{~cm}$ from the source with a $10 \mathrm{~cm}$ by $10 \mathrm{~cm}$ ficld. Measurements of the sensor output on a pulse-by-pulse basis were performed in order to study the signal characteristics and temporal response of the sensor as a function of the screen type and heam energy. The direct output signal from the sensor was amplificd and fed into a LeCroy 940)(A 8 bit digital oscilloscope. The waveform was read out and integrated over the region of interest with an appropriatc bascline subtraction. The number of signal electrons per pulse from the sensor was then calculated using a previously measured calibration coefficient linking the number of output electrons with the integration area. Since a minimum of $1 \mathrm{to} 2 \mathrm{cG}$ y is required to form a clinically useful image. the results of the pulsed measurements are presented as the number of signal electrons per unit dose in tahle 1 . These results have heen renormalized in give the number of measured signal electrons per high energy secondary

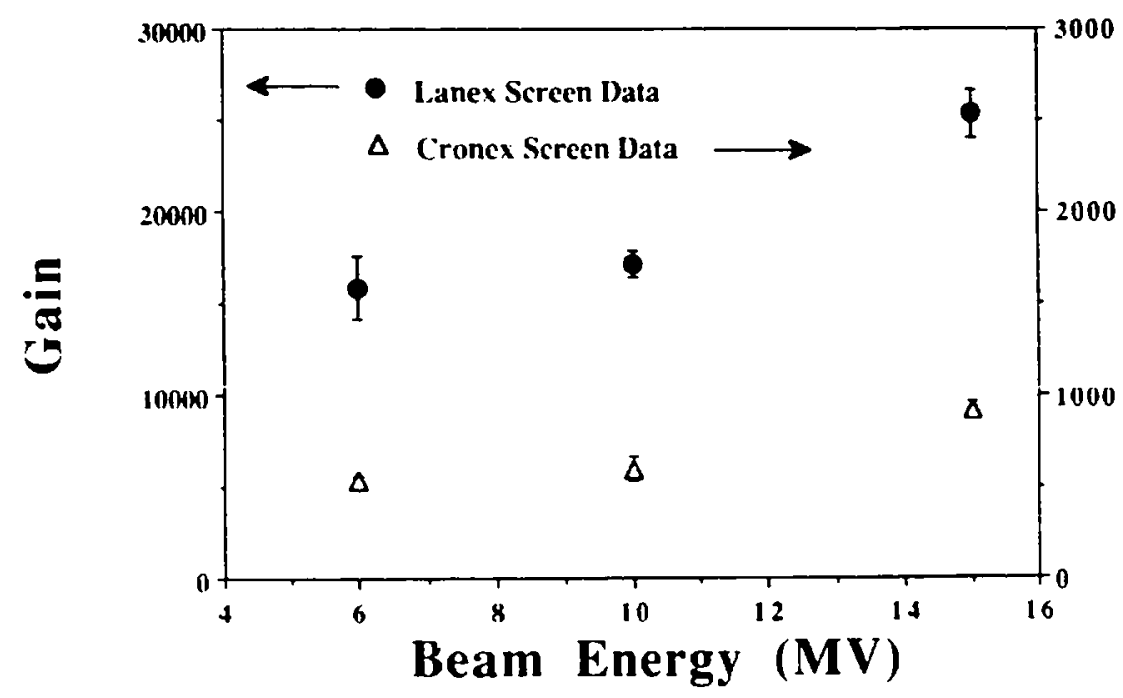

Fig. 5. Plot of the number of signal electrons measured from an a-Si:H sensor per high energy electron entering the phosphor screen as a function of screen lype and heam energy. 
Table 1

Summary of the number of signal electrons (in units of $10^{\prime \prime}$ ) meisured from the sensorr per unit dose as a function of screen type and beam energy. The results per pulse may be derived from the table. The values given in brackets are the standard deviations

\begin{tabular}{lllll}
\hline $\begin{array}{l}\text { Beum } \\
\text { energy } \\
{[\mathrm{MV}]}\end{array}$ & $\begin{array}{l}\text { Pulse } \\
\text { rate } \\
{[\mathrm{Hz}]}\end{array}$ & $\begin{array}{l}\text { Machine } \\
\text { output } \\
{[\mathrm{CGy} / \mathrm{min}]}\end{array}$ & $\begin{array}{l}\text { Signal electrons/cGy } \\
{\left[\times 10^{\mathrm{h}}\right]}\end{array}$ & $\begin{array}{l}\text { Cronex } \\
\text { Cincx }\end{array}$ \\
\hline 6 & 170 & 233 & $51(3)$ & $1528(175)$ \\
10 & 150 & 301 & $42(5)$ & $1198(48)$ \\
15 & 70 & 239 & $49(2)$ & $1356(70)$ \\
\hline
\end{tabular}

electron released from the metal plate using published Montc Carlo results $[10,14]$. These renormalized values are presented in fig. 5 , and they relate to the gain factors of the final four stages of a MASDA imager as shown in fig. 4. The Cronex and Lanex results demonstrate similar energy dependence. While the Cronex signals are $\sim \mathbf{3 0}$ times smaller than those for Lanex, its gain factor may still be sufficiently large to produce images of comparable quality. In addition, such $\mathrm{CaWO}_{4}$ screens are interesting since this phosphor decays in tens of microseconds compared to the $\sim 1 \mathrm{~ms}$ decay time of the $\mathrm{Gd}_{2} \mathrm{O}_{2} \mathrm{~S}: \mathrm{Tb}$. Such a short decay time could greatly facilitate cleaner extraction of the signals from the array sensors between pulses of ridiation.

\section{Discussion}

Techniques to allow the fabrication of denser and larger surfaces of a-Si: $\mathrm{H}$ devices for that panel displays are being vigorously pursued. Radiological imaging will benefit as a result. The maximum surface arca is most immediately limited by the size of the mask aligners used in the fabrication process. Currently, these allow the creation of arrays up to approximatcly $30 \mathrm{~cm}$ by 30 $\mathrm{cm}$ : expectations are that this linear dimension may double this decade. For radiotherapy imaging. arrays with $10^{5}$ to $10^{\prime \prime}$ pixcls with a pixcl pitch of $\sim 5(0) \mu \mathrm{m}$ are consistent with the imaging needs and with presently attainable yields and are the immediate goal of our rescarch. For diagnostic imaging considerably higher densitics are desirable. Current mask alignnent tolerances of $-5 \mu \mathrm{m}$ imply a limit of -100 to $150 \mu \mathrm{m}$ pixel pitch and thus $10^{11} 1010^{7}$ pixcls would be possible. However, denser arrays will suffer from large cross-over capacitance which degrades the signal-tonoisc ratio and readout speed, and considerable research will be necessary in order to achicve acceptable yields. The future development of MASDA-type imagers therefore presents both exciting research challenges and the prospect of improved imaging devices.

\section{Acknowledgements}

Support by a grant from the Whilaker foundation is gratcfully acknowledged. This work is also supported in part by National Institutes of Hcalth Grant No. 1-R01-CA51397-01. We wish to thank Mary Kay Witkowski for preparation of the figures.

\section{References}

[1] N.A. Baily et al.. Int. J. Radiat. Oncol. Biol. Phys. 6 (1980) 9.35 .

[2] J. Leong. Phys. Med. Biol. 31 (1986) 985.

[3] S. Shalev et al.. Comp. Med. Imag. Graphics 1.3 (1989) 217.

[4] A.G. Visser et al.. Int. J. Radiat. Oncol. Biol. Phys. 18 (199(1) 43.

[5] P. Munro et al.. Int. J. Radiat. Oncol. Biol. Physs. 18 $(199(1) 6+1$.

[6] J. Wong a al.. Int. J. Raddiat. Oncol. Biol. Phys. is (1990) 1477.

[7] M. van lterk and H. Meertens. Ratdiot. and Oncol. II (1988) 369 .

[8] K.S. Lam et al.. Br. J. Radiol. $59(1986) 10(17$.

[9] E. Morton et al.. Med. Phys. 18(4) (1991) 681.

[10] P. Munro et al.. SPIE Proc. Medical Imaging 111 (1989) 1090.

[11] L.E. Antonuk et al.. Nucl. Instr. and Meth. A299 (1990)) 14.3.

[12] I.D. French et al., Appl. Phys. A.31 (1983) 19.

[1.3] L.E. Antonuk et al.. IEEE Trans. Nucl. Sci. NS-37 (1990) 165.

[14] D.W.O. Rogers. Health Phys. 46 (1944) 891. 